\title{
Microsatellite marker variation in populations of the melon fly parasitoid, Psyttalia fletcheri
}

\author{
Somjit Homchan $^{\mathrm{a}}$, David S. Haymer ${ }^{\mathrm{b}}$, Sangvorn Kitthawee ${ }^{\mathrm{a}, *}$ \\ a Department of Biology, Faculty of Science, Mahidol University, Rama 6 Road, Bangkok 10400 Thailand \\ b Department of Cell and Molecular Biology, University of Hawaii at Manoa, Honolulu, HI, 96822, USA
}

*Corresponding author, e-mail: sangvorn.kit@mahidol.ac.th

\begin{abstract}
The parasitoid Psyttalia fletcheri (Silvestri) is an important natural enemy of the melon fly, Bactrocera cucurbitae (Coquillett). Melon fly infestations are responsible for extensive losses of cucurbit production worldwide, and P. fletcheri has been used for some time in biological control programmes attempting to deal with this pest. However, there is a general lack of knowledge of the genetic structure of populations of P. fletcheri, and the development of this information is key to the effective use of this parasitoid. In this study, we isolated several novel microsatellite loci to investigate the genetic structure of $P$. fletcheri populations from six locations in Thailand. All the loci analysed here were polymorphic, and the mean number of alleles per locus ranged from 4.2-8.6. Heterozygote deficiencies were noticed in most populations. Overall $F_{\mathrm{ST}}$ estimates showed moderate genetic differentiation among P. fletcheri populations with a jackknife mean of 0.084 . However, pairwise $F_{\mathrm{ST}}$ calculations revealed that 11 out of 15 population comparisons showed genetic differentiation. The greatest level of differentiation was also found for the population that had the lowest value for genetic diversity. In contrast, populations with high levels of genetic variation did not show significant genetic differentiation, nor did they show significant isolation by distance. An unrooted dendrogram constructed from Nei's genetic distance values also confirmed that one population from the south of Thailand can be separated from the others.
\end{abstract}

KEYWORDS: molecular markers, fruit fly parasitoid, genetic structure

\section{INTRODUCTION}

Psyttalia fletcheri (Silvestri) (Hymenoptera: Braconidae) is a parasitoid of fruit fly larvae that has been reported to attack the melon fly, Bactrocera cucurbitae (Coquillett) (Diptera: Tephritidae) ${ }^{1}$. This fruit fly is a serious pest of cucurbit plants in tropical Asia, Oceania, Indo-Pacific, and some parts of Africa ${ }^{2}$. Because of its parasitoid activity, P. fletcheri is considered to be an important potential biological control agent to aid in the reduction or elimination of melon fly populations. For example, it was introduced from south India to Hawaii in 1916, and it quickly became established as an effective biological control agent ${ }^{3,4}$.

Following this, P. fletcheri became a top candidate for many parasitoid-based melon fly control programmes $^{2}$. Its effectiveness, however, declined after a period of several years, and it was hypothesized that the mass rearing of $P$. fletcheri as a biological control agent may have led to a reduction in genetic variation due to population bottlenecks $\mathrm{s}^{3,4}$. This in turn was thought to have resulted in low fitness and diminished performance as a parasitoid ${ }^{5}$. However, the baseline data needed to test this hypothesis are sparse at best, particularly with regard to information about the levels of variation and the genetic structure of natural populations of this parasitoid.

In recent years several molecular markers have been developed for genetic studies of both fruit fly and parasitoid populations, specifically for the detection and study of genetic differentiation and for the estimation of genetic diversity within and among populations. Markers have been derived from mitochondrial and nuclear genes such as cytochrome oxidase $I(\mathrm{COI})^{6}$, the ribosomal internal transcribed spacer region 2 (ITS 2$)^{7}$, and $28 \mathrm{SDNA}^{8}$. Also diverse methods such as amplified fragment length polymorphisms ${ }^{9}$, single-stranded conformational polymorphisms ${ }^{10}$, and simple sequence repeats or microsatellites have been employed for these analyses ${ }^{11,12}$.

Among the markers and methods used, microsatellites have a number of advantages for population genetic analysis in that they tend to be highly polymorphic and exhibit co-dominant inheritance. Also, as with other DNA-based methods used in conjunction with the polymerase chain reaction (PCR), only small amounts of material are required ${ }^{13}$. For these and other reasons, microsatellite markers have been widely used for many studies of genetic diversity, analysis of parentage, and resolution of potential 
cryptic species complexes ${ }^{14}$, including the melon fly ${ }^{12}$. For parasitoid species in particular, in the past decade, microsatellite markers have been developed and used for population genetic studies of some parasitoid wasps such as Diaeretiella rapae (McIntosh) and Aphidius ervi Haliday ${ }^{15}$. For fruit fly parasitoids such as Psyttalia lounsburyi (Silvestri), Bon et al identified 21 polymorphic microsatellite loci and assessed genetic diversity in Kenyan and South African populations ${ }^{11}$. Cheyppe-Buchmann et al also used multiple loci to characterize the genetic diversity of $P$. lounsburyi populations ${ }^{16}$. Recently, the initial characterization of the genomes of three Nasonia parasitoid wasp species was announced ${ }^{17}$, and the distribution of microsatellites in these species has been analysed ${ }^{18}$.

Although the population genetic structure of the melon fly has been evaluated over a wide geographical range using various markers such as the mtDNA COI gene and microsatellites ${ }^{6,12}$, to our knowledge no studies reporting on the genetic structure of the melon fly parasitoid, P. fletcheri, have been published. To address this we have undertaken a study of microsatellite markers to analyse the genetic structure of this parasitoid in Thai populations.

The objectives of this study were to (1) develop microsatellite markers from the genome of $P$. fletcheri and (2) evaluate genetic variation within and among natural populations of this parasitoid collected from different locations in Thailand. An understanding of the genetic structure and variation within and among populations of this parasitoid is a critical component of the basic knowledge needed to effectively utilize it as a biological agent for control of the melon fly. The markers identified here may also provide useful tools for detecting any changes in population genetic structure occurring over time and for monitoring the performance of released parasitoids.

\section{MATERIALS AND METHODS}

\section{Sample collection}

Samples of $P$. fletcheri were obtained from collections of the ivy gourd, Coccinia grandis (L.) Voigt, infested with melon fly larvae in abandoned areas without insecticide treatment from six different locations in Thailand: Chumphon (CP), Chanthaburi (CT), Kanchanaburi (KN), Nonthaburi (NB), Nakhon Ratchasima (NR), and Phitsanulok (PS) (Table 1). We collected fruits infested with fruit fly larvae from each locality and transported them to the laboratory at Mahidol University, Bangkok, Thailand. Parasitoids were identified based on morphological characteristics
Table 1 Locations and sample sizes for $P$. fletcheri populations from Thailand used in this study.

\begin{tabular}{lrrc}
\hline Locality & Latitude & Longitude & Sample size \\
\hline Kanchanaburi & $14.2391^{\circ}$ & $99.0574^{\circ}$ & 64 \\
Chanthaburi & $12.6499^{\circ}$ & $102.0324^{\circ}$ & 63 \\
Nonthaburi & $13.8254^{\circ}$ & $100.4941^{\circ}$ & 79 \\
Nakhon Ratchasima & $14.7405^{\circ}$ & $102.3958^{\circ}$ & 60 \\
Chumphon & $9.7916^{\circ}$ & $98.7766^{\circ}$ & 19 \\
Phitsanulok & $16.7510^{\circ}$ & $100.1930^{\circ}$ & 44 \\
\hline
\end{tabular}

using the keys of Wharton and Gilstrap ${ }^{19}$. After identification, wasp specimens were kept at $-80^{\circ} \mathrm{C}$ for molecular genetic analysis.

\section{DNA isolation and microsatellite analysis}

Total genomic DNA was isolated from adult parasitoids following the Lifton protocol ${ }^{20}$. Microsatellites were isolated and characterized using $5^{\prime}$ anchored PCR primers ${ }^{21}$ and partial enriched library as modified from Hamilton et $\mathrm{al}^{22}$. Microsatellite primers were designed using PRIMER $3^{23}$. Each locus was tested for polymorphism in individual female parasitoids from field collections. From these, five polymorphic loci were chosen for analysis of genetic variation (Table 2).

Amplifications of microsatellite sequences were carried out in a total volume of $15 \mu$ l containing $10 \mathrm{ng}$ genomic DNA from a single female parasitoid, $1 \times$ PCR reaction buffer $(75 \mathrm{mM}$ Tris- $\mathrm{HCl}, \mathrm{pH} 8.0$, $20 \mathrm{mM}\left(\mathrm{NH}_{4}\right)_{2} \mathrm{SO}_{4}, 0.01 \%$ Tween 20$), 1.5 \mathrm{mM}$ $\mathrm{MgCl}_{2}, 0.2 \mathrm{mM}$ of each dNTP, $0.3 \mu \mathrm{M}$ of each primer, 0.5 U of Taq DNA polymerase (Fermentas). A PCR enhancer supplement of $1 \times$ MasterAmp PCR enhancer with betaine (Epicentre) was used for amplification of the Pftc12, Pftg15 and Pftg18 loci. The Pftca01 and Pftg26 loci were supplemented with $0.1 \mathrm{mg} / \mathrm{ml} \mathrm{BSA} \mathrm{(Sigma).} \mathrm{Amplification} \mathrm{reactions}$ were conducted in a Peltier based Thermal Cycler (MJ Research PTC-200). The amplification profile consisted of an initial denaturing step at $95{ }^{\circ} \mathrm{C}$ for 2 min (once); 25-30 cycles of $30 \mathrm{~s}$ denaturation at $94{ }^{\circ} \mathrm{C}, 30 \mathrm{~s}$ at $50-62{ }^{\circ} \mathrm{C}$ for annealing (Table 2), $30 \mathrm{~s}$ at $72{ }^{\circ} \mathrm{C}$ for new synthesis. A final extension was also done for $30 \mathrm{~min}$ at $72{ }^{\circ} \mathrm{C}$. The PCR products of each locus were analysed using electrophoresis on 6\% denatured polyacrylamide gels (Sequi-Gen GT sequencing Cell, BIO-RAD). A 10 bp ladder was used as a standard DNA size marker for determination of the size of individual amplified alleles. Alleles were visualized with the silver staining protocol described by Benbouza et $\mathrm{al}^{24}$. The stained plate was air dried 
Table 2 Primers and annealing temperatures used for amplification of five microsatellite loci. Number of alleles and allele size range tested in 329 females of $P$. fletcheri collected from six locations in Thailand.

\begin{tabular}{|c|c|c|c|c|c|c|}
\hline Locus & GenBank $^{\dagger}$ & Original clone $\mathrm{e}^{\ddagger}$ & Primer sequence $5^{\prime}$ to $3^{\prime}$ & Annealing temp & No. ${ }^{*}$ & Size range \\
\hline Pftca01 & JX026962 & $(\operatorname{tg})_{6},(\mathrm{tca})_{4}$ & $\begin{array}{l}\text { F: CATCGGTGTTTGAGGAAAAAA } \\
\text { R: GACCGAGTGTGTGTGTGTCA }\end{array}$ & $55^{\circ} \mathrm{C}$ & 2 & $179-181 \mathrm{bp}$ \\
\hline Pftc12 & JX026963 & $(\mathrm{tc})_{3} \operatorname{gg}(\mathrm{tc})_{5}$ & $\begin{array}{l}\text { F: CCGCATGAGGCAGTAAAGAA } \\
\text { R: GGCACTTCCTTGTCGCTTTA }\end{array}$ & $55^{\circ} \mathrm{C}$ & 13 & $180-220 \mathrm{bp}$ \\
\hline Pftg15 & JX026964 & $(\mathrm{gt})_{20}$ & $\begin{array}{l}\text { F: TCAAGGTCCTGCAGTTCTCAG } \\
\text { R: TCACTCTGTTGCAGCATAGGACTC }\end{array}$ & $62{ }^{\circ} \mathrm{C}$ & 14 & $124-152 \mathrm{bp}$ \\
\hline Pftg18 & JX026965 & $(\operatorname{tg})_{48}$ & $\begin{array}{l}\text { F: CCCAGGAGGAGTGCGTGTG } \\
\text { R: GAGGGTGGAGGGATTCTGCT }\end{array}$ & $62^{\circ} \mathrm{C}$ & 31 & $184-266 \mathrm{bp}$ \\
\hline Pftg26 & JX026966 & $(\mathrm{gt})_{9} \mathrm{n}_{10}(\mathrm{at})_{5}$ & $\begin{array}{l}\text { F: GGTGTGTGTGTGTGTGTGT } \\
\text { R: GGAGACTCAGTCATTCCCAAT }\end{array}$ & $50{ }^{\circ} \mathrm{C}$ & 4 & $186-192 \mathrm{bp}$ \\
\hline
\end{tabular}

${ }^{\dagger}$ GenBank accession number. ${ }^{\ddagger}$ Repeat motif of original clone. ${ }^{*}$ Number of alleles.

and photographed for genotyping, and allele sizes were estimated using image analysis software (TL100, TotalLab).

\section{Data analysis}

Tests for deviations from Hardy-Weinberg equilibrium (HWE) and genotypic disequilibrium were carried out using FSTAT 2.9.3 (www.unil.ch/popgen/ softwares/fstat.htm). The analysis of genetic diversity in $P$. fletcheri populations, including estimation of the mean number of alleles per locus $\left(N_{\mathrm{A}}\right)$, effective number of alleles $\left(N_{\mathrm{E}}\right)$, observed heterozygosity $\left(H_{\mathrm{O}}\right)$, and Nei's expected heterozygosity $\left(H_{\mathrm{E}}\right)^{25}$ were calculated using POPGENE 1.32 (www.ualberta.ca/ $\sim$ fyeh/). Global $F$-statistics over loci, including $F_{\text {IS }}$, $F_{\text {IT }}$ and $F_{\text {ST }}$, were calculated using FSTAT $^{26}$. Confidence intervals $(95 \% \mathrm{CI})$ for the $F$-statistics were estimated by bootstrapping over loci. Values for genetic differentiation between populations (pairwise $F_{\mathrm{ST}}$ ) were also examined using ARLEQUIN 3.1 ${ }^{27}$. Nei's genetic distance values between all pairs of populations $^{28}$ were calculated using POPGENE 1.32, and these estimates were used to construct a neighbourjoining dendrogram using TREEVIEW ${ }^{29}$. The correlation between geographical distance and genetic divergence between population pairs was tested using the Mantel test with 1000 permutations as implemented in GENEPOP 4.0.10 ${ }^{30}$.

\section{RESULTS}

\section{Isolation and characterization of microsatellite markers}

A total of 74 clones were picked for sequencing from two libraries constructed using the $5^{\prime}$ anchored PCR method and the modified enrichment library method. All sequenced clones contained microsatellite repeats shorter than 10 repeats in length, except for two showing continuous dinucleotide repeats of variable length $(\mathrm{GT})_{20}$ and $(\mathrm{TG})_{48}$. Fourteen microsatellite loci were chosen for further study.

Amplification and genotyping were done in individuals collected from natural populations. Of the 14 loci, five were found to be polymorphic when tested on a total of 329 P. fletcheri females. The number of alleles per locus ranged between 2 and 31 . Two loci (Pftca01 and Pftg26) showed relatively low allelic variation (2 and 4 alleles per locus, respectively) whereas the other three loci (Pftc12, Pftg15 and Pftg18) showed higher diversity at 13, 14, and 31 alleles per locus, respectively. The information specific to each locus is summarized in Table 2. No significant evidence for linkage disequilibrium was observed for these loci (after Bonferroni correction).

\section{Genetic variability within populations and HWE}

Genetic diversity of the $P$. fletcheri collections from six locations was investigated using five of the microsatellite loci identified here. The microsatellites used were polymorphic in all populations except for Pftca01 (fixed in the NB and NR populations) and Pftg26 (fixed in the PS population).

Deviations from HWE $(p<0.05)$ were observed in three populations (CT, NR, and PS). As shown in Table 3, in some cases significant homozygote excesses were observed. The NR population showed both the highest overall value of $F_{\text {IS }}(0.360)$ and the lowest frequency of heterozygotes $\left(H_{\mathrm{O}}=0.224\right)$.

Table 3 also shows that, the $\mathrm{CT}$ and $\mathrm{KN}$ populations showed the highest mean number of alleles per locus $\left(N_{\mathrm{A}}=8.6\right.$ and 7.2, respectively) and effective numbers of alleles ( $N_{\mathrm{E}}=5.20$ and 4.49 , respectively). A total of seven private alleles were also detected in the CT population, but only one was found for 
Table 3 Mean number of alleles per locus $\left(N_{\mathrm{A}}\right)$, effective number of alleles $\left(N_{\mathrm{E}}\right)$, private allele number $\left(P_{\mathrm{A}}\right)$, observed heterozygosity $\left(H_{\mathrm{O}}\right)$, expected heterozygosity $\left(H_{\mathrm{E}}\right)$, $F_{\text {IS }}$, and significant tests for HWE.

\begin{tabular}{lcccccccc}
\hline Pop. & Size & $N_{\mathrm{A}}$ & $N_{\mathrm{E}}$ & $P_{\mathrm{A}}$ & $H_{\mathrm{O}}$ & $H_{\mathrm{E}}$ & $F_{\mathrm{IS}}$ & $p^{\dagger}$ \\
\hline KN & 64 & 7.2 & 4.49 & 1 & 0.412 & 0.461 & 0.115 & 0.0020 \\
$\mathrm{CT}$ & 63 & 8.6 & 5.20 & 7 & 0.336 & 0.436 & $0.238^{*}$ & 0.0017 \\
$\mathrm{NB}$ & 79 & 4.6 & 1.56 & 0 & 0.225 & 0.219 & -0.022 & 0.8277 \\
$\mathrm{NR}$ & 60 & 6.4 & 2.99 & 3 & 0.224 & 0.345 & $0.360^{*}$ & 0.0017 \\
$\mathrm{CP}$ & 19 & 4.2 & 2.19 & 1 & 0.347 & 0.408 & 0.174 & 0.0186 \\
$\mathrm{PS}$ & 44 & 6.6 & 2.95 & 2 & 0.314 & 0.357 & $0.133^{*}$ & 0.0017 \\
\hline
\end{tabular}

$\dagger$ HWE $p$-value.

* Significance after Bonferroni correction (the critical level with Bonferroni correction is $p=0.0017$ ).

Table $4 F$-statistics values of overall population estimated by method of Weir and Cockerham ${ }^{26}$, including $F_{\text {IS }}, F_{\text {IT }}$ and $F_{\mathrm{ST}}$ values for five loci.

\begin{tabular}{lccc}
\hline Locus & $F_{\text {IS }}$ & $F_{\text {IT }}$ & $F_{\text {ST }}$ \\
\hline Pftca01 & -0.120 & 0.012 & 0.118 \\
Pftc12 & 0.074 & 0.140 & 0.071 \\
Pftg15 & 0.264 & 0.312 & 0.065 \\
Pftg18 & 0.212 & 0.285 & 0.092 \\
Pftg26 & -0.008 & 0.022 & 0.029 \\
Mean $^{\dagger}$ & 0.187 & 0.255 & 0.084 \\
95\% CI & $-0.001-0.227$ & $0.077-0.287$ & $0.063-0.093$ \\
\hline
\end{tabular}

$\dagger$ The jackknife mean and the $95 \%$ confidence intervals (CI) values were determined by bootstrapping over all loci.

$\mathrm{KN}$. The lowest within-population genetic diversity value $\left(F_{\mathrm{IS}}\right)$ was observed in the NB population. This population had a mean number of allele 4.6 alleles per locus, an effective number of alleles of 1.56 , and no private alleles. In addition, the expected heterozygosity $\left(H_{\mathrm{E}}=0.219\right)$ for this population was the lowest among the estimates obtained here.

Hierarchical $F$-statistics, $F_{\text {IS }}, F_{\text {IT }}$ and $F_{\text {ST }}$ were also estimated for all of the populations (Table 4). Over all loci the mean value of $F_{\mathrm{IS}}$ was positive, but not significantly different from zero $\left(F_{\mathrm{IS}}=0.187\right.$, 95\% confidence interval (CI): $-0.001-0.227)$. The mean values for both $F_{\mathrm{IT}}$ and $F_{\mathrm{ST}}$ were significantly different based on their 95\% CIs, with jackknife mean values of 0.255 and 0.084 , respectively.

\section{Genetic differentiation among populations of P. fletcheri}

In terms of population differentiation, after Bonferroni corrections, 11 of the 15 pairwise $F_{\mathrm{ST}}$ comparisons were significantly different (Table 5). The highest
Table 5 Matrix of pairwise $F_{\mathrm{ST}}$ over five microsatellite loci among populations (above diagonal) and Nei's ${ }^{28}$ unbiased genetic distance ( $D$, below diagonal).

\begin{tabular}{lcccccc}
\hline Pop. & KN & CT & NB & NR & CP & PS \\
\hline KN & - & 0.0169 & $0.1325^{*}$ & $0.0417^{*}$ & 0.0761 & 0.0255 \\
CT & 0.0171 & - & $0.1248^{*}$ & $0.0241^{*}$ & 0.0819 & $0.0274^{*}$ \\
NB & 0.0689 & 0.0640 & - & $0.1398^{*}$ & $0.2429^{*}$ & $0.1314^{*}$ \\
NR & 0.0289 & 0.0170 & 0.0661 & - & $0.1269^{*}$ & $0.0303^{*}$ \\
CP & 0.0750 & 0.0759 & 0.1220 & 0.0809 & - & $0.1448^{*}$ \\
PS & 0.0185 & 0.0205 & 0.0598 & 0.0207 & 0.1154 & - \\
\hline
\end{tabular}

* Values of $F_{\mathrm{ST}}$ significantly different from zero (after Bonferroni corrections).

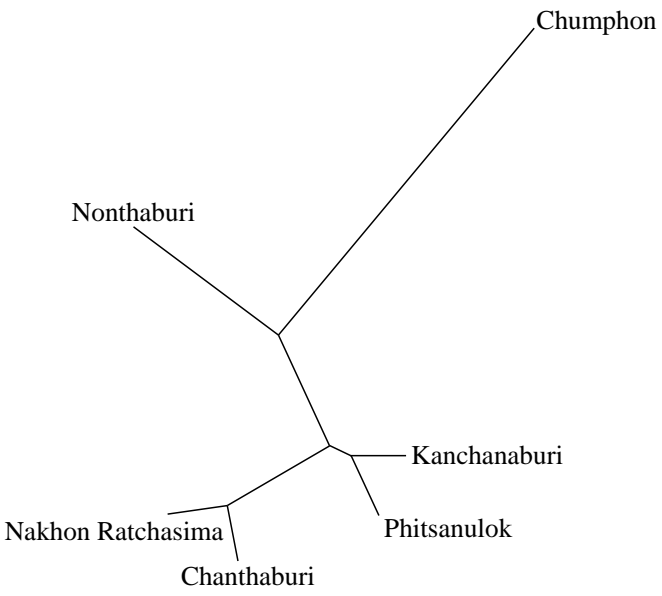

$\underline{0.5}$

Fig. 1 Unrooted dendrogram illustrating genetic relationships among six populations of P. fletcheri based on Nei's unbiased genetic distance.

$F_{\mathrm{ST}}$ value $(0.2429, p<0.05)$ was found in the comparison between the $\mathrm{NB}$ and $\mathrm{CP}$ populations, while the lowest pairwise value for $F_{\mathrm{ST}}(0.0169, p=0.30)$ was observed between the $\mathrm{KN}$ and $\mathrm{CT}$ populations.

Table 5 also shows that the Nei genetic distance values among the $P$. fletcheri populations analysed here ranged from $0.0170-0.1220$. The distance was greatest between the NB and $\mathrm{CP}$ populations $(D=$ 0.1220 ) and lowest between the CT and NR populations $(D=0.0170)$. The unrooted dendrogram constructed based on these unbiased Nei's genetic distance values ${ }^{28}$ showed no obvious overall pattern of clustering of populations (Fig. 1), but the $\mathrm{CP}$ and NB populations from the south and central regions, respectively, do appear to be separated from the others. Significant relationships between geographical distance and pairwise $F_{\mathrm{ST}}(p=0.420)$ or Nei's genetic distance ( $p=0.162)$ using the Mantel test with 1000 permutations were not found. 


\section{DISCUSSION}

\section{Genetic variation and differentiation in natural populations of $P$. fletcheri}

In this study using microsatellite markers, we found mean values for heterozygosity ranging between 0.219 and 0.461 in six wild populations of P. fletcheri. This is considerably higher compared to the values found using protein markers found for other haplodiploid systems including parasitoid wasp species. For solitary wasps, for example, the mean allozyme heterozygosities were found to range between 0.024 and $0.067^{31}$. A similar study of six populations of the fruit fly parasitoid, Diachasmimorpha longicaudata (Ashmead), had values between 0.119 and $0.358^{32}$.

Other studies have reported similar, relatively high mean heterozygosity values for microsatellite markers, such as values ranging from $0.280-0.641$ for four loci in populations of the aphid parasitoid D. rapae ${ }^{15}$. Other examples from insect studies using microsatellites where even higher values were obtained include mean heterozygosities of 0.614 obtained for seven loci in the honeybee Apis mellifera $\mathrm{L}^{33}$, and 0.729 for three loci in three species of ants ${ }^{34}$. The extent to which these differences can be attributed to the different organisms versus the different marker systems is yet to be resolved.

In terms of individual populations of $P$. fletcheri, we found relatively low levels of genetic variation in the NB population even though there was no significant deviation from HWE nor any signs of homozygote excess $\left(F_{\mathrm{IS}}=-0.022\right)$. In this population, the total number of alleles here was relatively low and no private alleles were found. Several factors may plausibly explain the differences in genetic structure seen for this population. This sample was collected from a patch surrounded by urban areas near Bangkok, while other samples analysed here were collected from undisturbed natural environments. Also at the collection site of this population, landscape changes or other environmental disturbances potentially impacting both host plants and host flies in this area may have produced a bottleneck event ${ }^{31}$. Overall, it appears that both genetic drift and ecological factors may have played important roles in reducing the genetic diversity and altering the genetic structure of this relatively isolated population.

Higher levels of genetic variation were found within the $\mathrm{KN}$ and CT populations. These same populations also had higher mean numbers of alleles per locus, as is typical for more variable populations ${ }^{35}$. These populations also appeared to be more homogeneous ( $98 \%$ or higher) in terms of genetic identity, and showed relatively little genetic differentiation from each other. The same phenomenon has been found among local populations of species of Drosophila willistoni Sturtevant where the mean value for genetic identity was relatively high at $97 \%^{36}$.

Three populations of $P$. fletcheri showed significant heterozygote deficiencies, especially the NR population where the $F_{\mathrm{IS}}$ value was 0.360 . Using protein polymorphisms, significant heterozygote deficiencies have also been reported previously in natural populations of $D$. longicaudata, a parasitoid of the oriental fruit fly, B. dorsalis (Hendel) ${ }^{32}$. A number of causal factors such as inbreeding and natural selection against heterozygotes may contribute to these heterozygote deficiencies. Lester and Selander ${ }^{31}$ also noted that heterozygote deficiency was typically found among many haplodiploid insects.

\section{Genetic distances and relationships among populations}

Across all investigated populations, the average $F_{\mathrm{ST}}$ value of 0.084 found here was significantly different from zero (based on the 95\% CI) and is somewhat similar to that reported in natural populations of D. longicaudata $\left(F_{\mathrm{ST}}=0.064\right)$ estimated from isozyme polymorphisms ${ }^{32}$. Between populations, 11 out of 15 of the pairwise $F_{\mathrm{ST}}$ comparisons made here were significant. These results suggest that there is only moderate genetic differentiation among populations of $P$. fletcheri, and this may be consistent with the idea that gene flow is still occurring among these populations. In contrast, a recent report on populations of $P$. lounsburyi, a parasitoid of the olive fruit fly Bactrocera oleae (Rossi) which used eight microsatellite loci and one mitochondrial locus, showed evidence of extensive genetic structuring over its entire geographical range in Africa ${ }^{16}$.

Beyond these studies, however, the data currently available on genetic variation and structure in natural populations of fruit fly parasitoids using microsatellite markers are limited. Population genetic studies of other parasitoids, such as the butterfly parasitoids Cotesia melitaearum (Wilkinson) and Hyposoter horticola (Gravenhorst) have been analysed using limited numbers of microsatellite loci ${ }^{37}$. The overall value for $F_{\mathrm{ST}}$ obtained for $C$. melitaearum $\left(F_{\mathrm{ST}}=0.378\right)$ was substantially greater than that for $H$. horticola $\left(F_{\mathrm{ST}}=0.063\right)$, suggesting considerable differences in the extent of genetic differentiation in these populations. Both species also showed significant results for pairwise $F_{\mathrm{ST}}$ comparisons and for geographical distance values ${ }^{37}$. Based on the results of the Mantel test conducted here, however, we did not see similar 
relationships for the populations of $P$. fletcheri.

Overall, the $P$. fletcheri populations we analysed appear to be relatively homogeneous. Values for Nei's genetic distance $(D)^{28}$ between populations ranged between $2 \%(\mathrm{KN}-\mathrm{CT})$ and $12 \%$ (NB-CP). The unrooted dendrogram illustrated no distinctive clustering (Fig. 1), although the CP population from the south showed the greatest distance from the others. Brussard et $\mathrm{al}^{38}$ noted that for insects, values for Nei's genetic distance among different populations of the same species tend to be in the range from $0 \%$ to $22 \%$. At other taxonomic levels, such as subspecies or sibling species, the distance values will tend to be higher. The P. fletcheri populations studied here all show levels of genetic differentiation well under $22 \%$.

To provide a clearer picture of the extent to which other factors including selection, drift, and inbreeding effects may also be operating on these populations, future studies of the genetic variability within this parasitoid species should be carried out over a larger geographical area using more markers.

In conclusion, this is the first report on the genetic structure of P. fletcheri populations using microsatellite markers. The results obtained may provide valuable information about genetic variation in natural populations in such parasitoids, and may provide a baseline set of data for comparison to document any loss of genetic variation when wild strains are brought into the laboratory for mass rearing. This phenomenon has been seen in other insect species ${ }^{39}$, and this loss of variability has major implications for biological control programmes that require mass rearing. Knowledge of the genetic structure of such natural populations may also be important for understanding how to preserve natural levels of genetic variation and to increase the effectiveness of the parasitoids as control agents.

Acknowledgements: We would like to thank Donald McInnis of the USDA Facility at Manoa, Honolulu, Hawaii for providing parasitoid samples for microsatellite isolation and Urasri Suyasunanont for helping with sample collection around the country. The financial support was provided by the Thailand Research Fund and the Commission on Higher Education Staff Development Project and Faculty of Science, Mahidol University. We also wish to thank the three anonymous reviewers for helpful comments.

\section{REFERENCES}

1. Chinajariyawong A, Clarke AR, Jirasurat M, Kritsaneepiboon S, Lahey HA, Vijaysegaran S, Walter GH (2000) Survey of opiine parasitoids of fruit flies (Diptera: Tephritidae) in Thailand and Malaysia. Raffles Bull Zool 48, 71-101.
2. Dhillon MK, Singh R, Naresh JS, Sharma HC (2005) The melon fruit fly, Bactrocera cucurbitae: A review of its biology and management. J Insect Sci 5, 40.

3. Clausen CP, Clancy DW, Chock QC (1965) Biological control of the oriental fruit fly (Dacus dorsalis Hendel) and other fruit flies in Hawaii. USDA, ARS, Technical Bulletin 1322.

4. Nishida T (1956) An experimental study of the ovipositional behavior of Opius fletcheri Silvestri (Hymenoptera: Braconidae), a parasite of the melon fly. Proc Hawaii Entomol Soc 16, 126-34.

5. Hufbauer RA, Roderick GK (2005) Microevolution in biological control: Mechanisms, patterns, and processes. Biol Contr 35, 227-39.

6. Prabhakar CS, Mehta PK, Sood P, Singh SK, Sharma P, Sharma PN (2012) Population genetic structure of the melon fly, Bactrocera cucurbitae (Coquillett) (Diptera: Tephritidae) base on mitochondrial cytochrome oxidase (COI) gene sequences. Genetica 140, 83-91.

7. Kitthawee S (2013) ITS2 sequence variations among members of Diachasmimorpha longicaudata complex (Hymenoptera: Braconidae) in Thailand. J Asia Pac Entomol 16, 173-9.

8. Rugman-Jones PF, Wharton R, Noort TV, Stouthamer R (2009) Molecular differentiation of the Psyttalia concolor (Szépligeti) species complex (Hymenoptera: Braconidae) associated with olive fly, Bactrocera oleae (Rossi) (Diptera: Tephritidae), in Africa. Biol Contr 49, $17-26$.

9. Kakouli-Duarte T, Casey DG, Burnell AM (2001) Development of a diagnostic DNA probe for the fruit flies Ceratitis capitata and Ceratitis rosa (Diptera: Tephritidae) using amplified fragment-length polymorphism. J Econ Entomol 94, 989-97.

10. Antolin MF, Ode PJ, Heimpel GE, O'Hara RB, Strand MR (2003) Population structure, mating system, and sex-determining allele diversity of the parasitoid wasp Habrobracon hebetor. Heredity 91, 373-81.

11. Bon MC, Jones W, Hurard C, Loiseau A, Ris N, Pickett C, Estoup A, Fauvergue X (2008) Identification of 21 polymorphic microsatellites in the African parasitoid wasp, Psyttalia lounsburyi (Silvestri) (Hymenoptera: Braconidae). Mol Ecol Resour 8, 930-2.

12. Wu Y, Li Z, Wu J (2009) Polymorphic microsatellite markers in the melon fruit fly, Bactrocera cucurbitae (Coquillett) (Diptera: Tephritidae). Mol Ecol Resour 9, 1404-6.

13. Tautz D (1989) Hypervariability of simple sequences as a general source for polymorphic DNA markers. Nucleic Acids Res 17, 6463-71.

14. Estoup A, Garnery L, Solignac M, Cornuet JM (1995) Microsatellite variation in honey bee (Apis mellifera L.) populations: hierarchical genetic structure and test of the infinite allele and stepwise mutation models. Genetics 140, 679-95.

15. Baker DA, Loxdale HD, Edwards OR (2003) Genetic variation and founder effects in the parasitoid wasp, 
Diaeretiella rapae (M'intosh) (Hymenoptera: Braconidae: Aphidiidae), affecting its potential as a biological control agent. Mol Ecol 12, 3303-11.

16. Cheyppe-Buchmann S, Bon MC, Warot S, Jones W, Malausa T, Fauvergue X, Ris N (2011) Molecular characterization of Psyttalia lounsburyi, a candidate biocontrol agent of the olive fruit fly, and its Wolbachia symbionts as a pre-requisite for future intraspecific hybridization. BioControl 56, 713-24.

17. Werren JH, Richards S, Desjardins CA, Niehuis O, Gadau J, Colbourne JK, Beukeboom LW, Desplan C, et al (2010) Functional and evolutionary insights from the genomes of three parasitoid Nasonia species. Science 327, 343-8.

18. Pannebakker BA, Niehuis O, Hedley A, Gadau J, Shuker DM (2010) The distribution of microsatellites in the Nasonia parasitoid wasp genome. Insect Mol Biol 19, 91-8.

19. Wharton RA, Gilstrap FE (1983) Key to and status of opiine braconid (Hymenoptera) parasitoids used in biological control of Ceratitis and Dacus s. 1. (Diptera: Tephritidae). Ann Entomol Soc Am 76, 721-42.

20. Anleitner JE, Haymer DS (1992) $Y$ enriched and $Y$ specific DNA sequences from the genome of the Mediterranean fruit fly, Ceratitis capitata. Chromosoma 101, 271-8.

21. Fisher PJ, Gardner RC, Richardson TE (1996) Single locus microsatellites isolated using $5^{\prime}$ anchored PCR. Nucleic Acids Res 24, 4369-71.

22. Hamilton MB, Pincus EL, Di Fiore A, Fleischer RC (1999) Universal linker and ligation procedures for construction of genomic DNA libraries enriched for microsatellites. BioTechniques 27, 500-7.

23. Rozen S, Skaletsky H (1999) Primer3 on the WWW for general users and for biologist programmers. In: Misener S, Krawetz SA (eds) Bioinformatics Methods and Protocols, pp 365-86.

24. Benbouza H, Jacquemin JM, Baudoin JP, Mergeai G (2006) Optimization of a reliable, fast, cheap and sensitive silver staining method to detect SSR markers in polyacrylamide gels. Biotechnol Agron Soc Environ 10, 77-81.

25. Nei M (1973) Analysis of gene diversity in subdivided populations. Proc Natl Acad Sci USA 70, 3321-3.

26. Weir BS, Cockerham CC (1984) Estimating $F$-statistics for the analysis of population structure. Evolution 38, 1358-70.

27. Excoffier L, Laval G, Schneider S (2005) Arlequin (version 3.0): An integrated software package for population genetics data analysis. Evol Bioinformat Online 1, 47-50.

28. Nei M (1978) Estimation of average heterozygosity and genetic distance from a small number of individuals. Genetics 89, 583-90.

29. Page RDM (1996) TreeView: An application to display phylogenetic trees on personal computers. Comput Appl Biosci 12, 357-8.
30. Raymond M, Rousset F (1995) GENEPOP (version 1.2): Population genetics software for exact tests and ecumenicism. J Hered 86, 248-9.

31. Lester LJ, Selander RK (1979) Population genetics of haplodiploid insects. Genetics 92, 1329-45.

32. Kitthawee S, Julsilikul D, Sharpe RG, Baimai V (1999) Protein polymorphism in natural populations of $\mathrm{Di}$ achasmimorpha longicaudata (Hymenoptera: Braconidae) in Thailand. Genetica 105, 125-31.

33. Estoup A, Scholl A, Pouvreau A, Solignac M (1995) Monoandry and polyandry in bumble bees (Hymenoptera; Bombinae) as evidenced by highly variable microsatellites. Mol Ecol 4, 89-94.

34. Hedrick PW, Parker JD (1997) Evolutionary genetics and genetic variation of haplodiploids and X-linked genes. Annu Rev Ecol Systemat 28, 55-83.

35. Lowe AJ, Moule C, Trick M, Edwards KJ (2004) Efficient large-scale development of microsatellites for marker and mapping applications in Brassica crop species. Theor Appl Genet 108, 1103-12.

36. Avise JC (1976) Genetic differentiation during speciation. In: Ayala FJ (ed) Molecular Evolution. Sinauer, Sunderland, MA, pp 106-22.

37. Kankare M, van Nouhuys S, Gaggiotti O, Hanski I (2005) Metapopulation genetic structure of two coexisting parasitoids of the Glanville fritillary butterfly. Oecologia 143, 77-84.

38. Brussard PF, Ehrlich PR, Murphy DD, Wilcox BA, Wright J (1985) Genetic distances and the taxonomy of checkerspot butterflies (Nymphalidae: Nymphalinae). J Kansas Entomol Soc 58, 403-12.

39. Haymer DS (1995) Genetic analysis of laboratory and wild strains of the melon fly (Diptera: Tephritidae) using random amplified polymorphic DNA-polymerase chain reaction. Ann Entomol Soc Am 88, 705-10. 